\title{
Modeling Extenics Innovation Software by Intelligent Service Components
}

\author{
Rui Fan*
}

School of Software, Guangdong Ocean University, Zhanjiang, China, 524008

\begin{abstract}
Independent innovation is the core of the national development strategy. After twenty years, Extenics made great progress in innovation theory, formal method and real application. Developing and manufacturing innovation software to help people develop intelligence, inspire creation is key for Extenics further developing. Now software model cannot meet the requirements of flexible alternate in innovation activities. To address this challenge, we propose to fusion agent, service and component for building autonomy, dynamic evolved, intelligent accumulated, great granularity intelligent service component (ISC), provide the good software entity for realization extension flexible thought. We try to expand extensible analysis, extension transformation, extension evaluation, dependent function and so on to services, according to extension innovation method dynamic integrate them into extension intelligent service system (EISS), finally establish a wise extension innovation software model. For software modeling provides a new way.
\end{abstract}

Keywords: Extenics Innovation Software, Extenics Intelligent Service System, Intelligent Service Component.

\section{INTRODUCTION}

To improve independent innovation ability, building an innovative country is the core of national development strategies. In the past two decades, Extenics has developed into a new discipline with a relatively mature theoretical framework. It made great progress for creativity and innovation in theory, formal methods and practical application. It describes the goals and conditions of the contradictory problems using formal methods, and models the nuclear problems. It uses flexible approach to extension analysis, and mines ideas, knowledge and way of solving problems by Extenics transformation, and ultimately educes creative and innovative strategies to meet user needs.

To generating high quality creative or innovative policy is not an easy task. Study simulating software about Extenics' flexible thought of "change incompatible to compatibility", "change confrontation to coexistence" will become key issues that must be solved. Some extension software has be developed, but to help businesses develop smart, inspired the extension of decision making, generate good ideas, stimulate innovative software still does not appear. Developing of Extenics innovative software is difficulties and challenges: 1) Extenics theory and methods still is in the initial stages of development, what kind of software model to support and enable creativity and innovation this flexible intelligence research has only just begun. 2) Existing software models focus on software evolution process match between entities, not involved the continuous evolution in the creative activities within the software entity and entities with environment caused by of complex problems.

\footnotetext{
*Address correspondence to this author at the School of Software, Guangdong Ocean University, Zhanjiang, China, 524008;

Tel: +86-13828258036; E-mail: fanrui@gdou.edu.cn
}

\section{RELATIVE WORKS}

Extenics researches the possibility of matters' extension and new rules and methods of innovation with formalized models, which are to be used to solve contradictions. The first paper [1], published in 1983, after more than 20 years of development, extenics theory, formal methods, engineering application have made great progress [2-5]. It describes the goals and conditions of the contradictions problem using formal methods, and models core model, in flexible ways to analysis possibility of matters' extension, leads good ideas, knowledge and way of solving contradictions problems by combining quantitative and qualitative transformation methods, ultimately generates creative or innovative policy. With the Extenics thought of "change infeasible to feasible", and "change incompatible to compatible", and "change opposing to coexistence", create out some Entenisc innovation methods e.g. Extenics data mining, diamond thinking, conversion bridge, Extenics policy generating and so on, and success to application to decision-making, control, diagnosis, evaluation and recognition, area [6-11], recent patents on Extenics software [12] also used to solve actual problem,such as Extenics strategy generation, Extenics data mining, Extenics design, Extenics control,and Extenics detecting and so on.

Product concept design method based on extension information matter-element (ZL200510062040.X)[13] discloses a production concept design method based on extendable information element in the production concept design domain. The invention displays good flexibility, rational mapping method, strong evolution ability and high inference efficiency.

Dummy assembly method based on extension-able correlation relation (ZL200810062525.2) [14] discloses a virtual assembly method based on the extension relationship. 
The invention effectively realizes quantitative assembly management in the presence of assembly error, and has strong practicality.

Part classification method based on developable clustering (ZL200810062348.8)[15] provides a part classification method based on the expansible cluster, which has clear relevancy analysis, high classification efficiency and high accuracy.

Non-current transformer type power-frequency weakcurrent electromagnetism signal sensor (ZL200920054202.9) [16] provides a utility model relates to a non-current transformer type power-frequency weak-current electromagnetism signal sensor. The electromagnetism signal sensor is simple, can be used easily and can be operated without any professional knowledge; the structure is simple, firm and wearable; the operation is safe and reliable; no radial, ultra-wave and the like can be released; and instruments do not need to be installed on the wire; therefore, the non-current transformer type power-frequency weak-current electromagnetism signal sensor is convenient and practical.

Indoor wall-embedded wire nondestructive detector (ZL200920054205.2)[17] provides a utility model relates to an indoor wall-embedded wire nondestructive detector. The detector is simple, can be used easily and can be operated without any professional knowledge; the structure is simple, firm and wearable; the operation is safe and reliable; no radial, ultra-wave and the like can be released; and instruments do not need to be installed on the wire; therefore, the indoor wall-embedded wire nondestructive detector is convenient and practical.

Automatic signing machine (ZL200920056631.X) [18] provides a utility model relates to an automatic signing machine, especially to a signing mechanism. The utility model provides an automatic signing machine suitable for various seals with different shapes. According to the utility model, the automatic signing machine can be suitable for various seals with different shapes, and the seal can be replaced conveniently.

Intelligent lighting control method for park landscapes based on RFID (radio frequency identification) and extension correlation functions (CN201010594980.4)[19] discloses an intelligent lighting control method for park landscapes based on RFID (radio frequency identification) and extension correlation functions. The reliability of the visitor flow rate detection result of the park is good, and the working conditions of the landscape lighting are adjusted in real time according to the visitor flow rate.

Method for resolving attribute conflicts in green design based on transforming bridge (CN201010160796)[20] provides a method for resolving attribute conflicts in green design based on a transforming bridge. The method is used for reasonably and effectively resolving the conflicts by using mathematical expression.

However, these Extenics software tackle specific domain problems. Help businesses develop smart, supports decision, generate good ideas, stimulate innovative and create intelligent software system does not appear. This affected the extension method in a variety of application, also constrain the further development of the theory and method of Extenics. To research Extension innovation software, must establish its software model. To describe large and complex software architecture, computer scientists in the development of large software systems is in the gradual deepening of understanding and development of software systems [21]. Industry introduced Darwin, Wright, ACME, A-ADL, $\mathrm{XYZ/ADL}$ Tracer and ABC/ADL software architecture description language, and uses the CRAM [22], Z language [23], Pi calculations [24-26], Bigraph [27-30], category theory [31-33] and software formal methods [34] to accurately describe software architecture. With the popularization of the Internet, network software [35-39] with open, dynamic evolution characteristics of self-government. But these formal methods focus on modeling and adaptation to change how to change structures and ensure the consistency of behavior, haven't resolve software conflicts with environmental or failing to conform to changing requirements. With autonomy, responsiveness, dynamism, social characteristics, agent may be new solution to lack of dynamic and intelligent of software architecture. Agentbased software engineering (AOSE) has been many studies [40-45]. Agent technology in advanced manufacturing field had been plenty application [46], implementing web services based on service agent also has research and application [47]. However, agent also does not support the extension construction of an innovative software model.

\section{INTELLIGENT SERVICE COMPONENT}

The intelligent service component (abbreviated ISC) model is as shown in (Fig. 1). By fusing benefits of agents, services and components, form a large granularity proactive intelligent service component. It is consists of intelligent reasoning centre and service container. Intelligent service components are differs from the BDI model:

1. By perception form goal: intelligent reasoning center percepts (Sensors) to outside environment or changes in user demand, form event queue by decision-making reasoning (Reasoning\&Decision-Making).Inference decision based on the nature of the incident and the mental states (BeliefsDB) analysis of semantic changes in event semantics form the initial demand, and thus achieve functional requirements and non-functional requirements of the goal tree to the goals library (GoalsDB). Environment or users needs change may want to change the facts (BeliefsDB), and lead to new goals to the goals library (GoalsDB). Some internal events (dashed arrow) also requires decision-making reasoning build new goals and match the appropriate subset of the plans for adapting the environment or user' changes. These plans (PlansDB) want to accomplish the goals for adapting to dynamic evolution of ISCs.

2. Mapping goals tree into the service tree: service container is intelligence gathering centre of services. According to goals layer-by-layer decomposition tree want to find service implemented subgoal and aggregate into corresponding service tree (Goals to Service). For each service on the service tree, may already has a provide service 


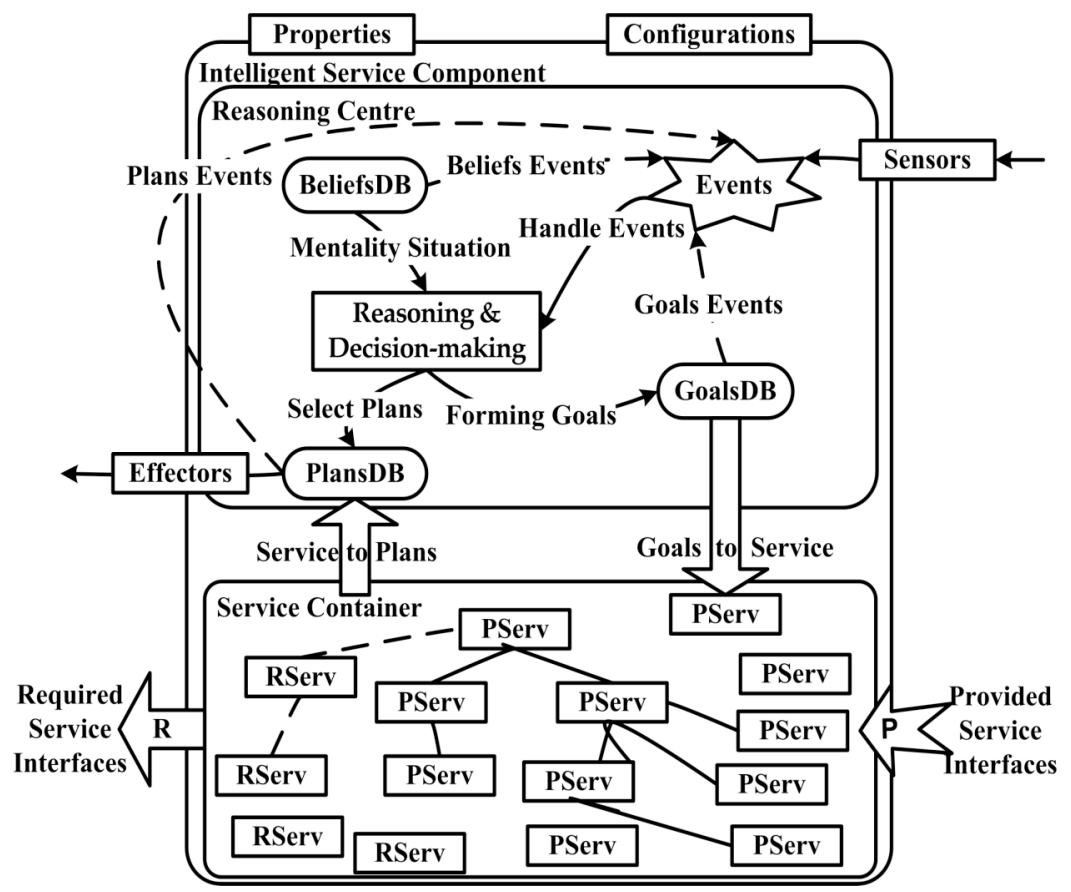

Fig. (1). Inteligent service component model.

(PServ) or must require an external service (RServ). Intelligent service component on-demand dynamic evolution are only two core actions: increase a service node or remove a service node, other actions are implemented by a combination of both actions (for example, service node is replacement by remove the old service node and add a new service node). Increase service is two states: one is by purchasing and downloading external services to the local adding the service node (PServ). Another is by requesting external services return results to local adding the service node (RServ and dotted line connection). With provided services and request service aggregation mechanism, multiple intelligence components synergy to accomplish more complex goals. Buy permanent service or rental temporary service for realizing intelligent service system depends on the economy and the importance of balance.

3. Packaging service tree generation plans: service container encapsulates the service tree into plans (Service to Plans) is performed to updating plan library for completing responses to environmental changes or user changes in demand.

\section{EXTENICS INTELLIGENT SERVICE COMPONENT}

The Extenics Intelligent Service Component is actually an intelligent services component based on extenics innovation elements framework. It has intelligent service components' autonomy, responsiveness, dynamism, social, also has Extenics elements' extensibility, variability, and flexibility features. Extension and transformation is the core of extenics intelligence. Extension involves divergence, correlative, implication, opening up and conjugation reasoning, which BDI model does not have these reasoning; transformation involves increasing/decreasing, expansion/ contraction, replacement, decomposition and duplication transforming, which BDI model does not have this kind of change mechanism. Expansion and transformation mechanism will greatly enrich, refine ISC cognitive capability and quantitative analysis reasoning can resolve conflicts problems effectively and adaptability. Intelligent service component can be customized separately into the basic-element intelligent service component, compositeelement intelligent service component. On this basis, respectively increasing dependent function service, Extenics transforms service, extension analysis service, Entenisc evaluation services and so on, we get a different function extension of Entensic intelligent service component (EISC), in other words, intelligent service in the service tree is the key.

\subsection{Basic Element Intelligent Service Component}

Basic element intelligent service component (BISC) includes matter-element (MatterElement), affair-element (AffairElement) and relation-element (RelationElement), is to describe things, acts or its associate. BISC has names (ElementName), one or more attributes and corresponding values. Such as the name, attributes, and attribute values can be stored in a relational database through database management system and connection with the basic element intelligent service components' beliefbase.

\subsection{Composite Element Intelligent Service Component}

Composite intelligent service component (CISC) Composite elements' attribute value may be either a basic element. As with ordinary basic element as, composite element such as the name, attributes, and attribute values can be stored in a relational database and connected with the composite set of beliefbase. By specific different attributes, attribute value, database content can be customized.

\subsection{Relation Function Service Tree}

Relation function services are mainly provided to the basic element intelligent service component, the composite 


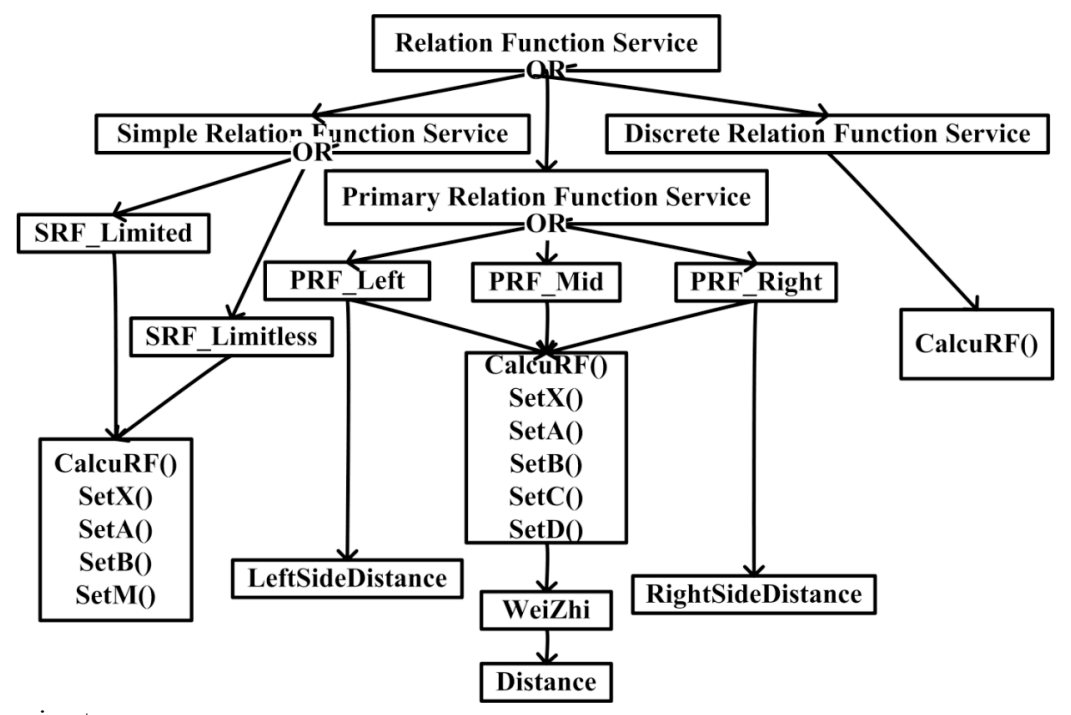

Fig. (2). Relation function service tree.

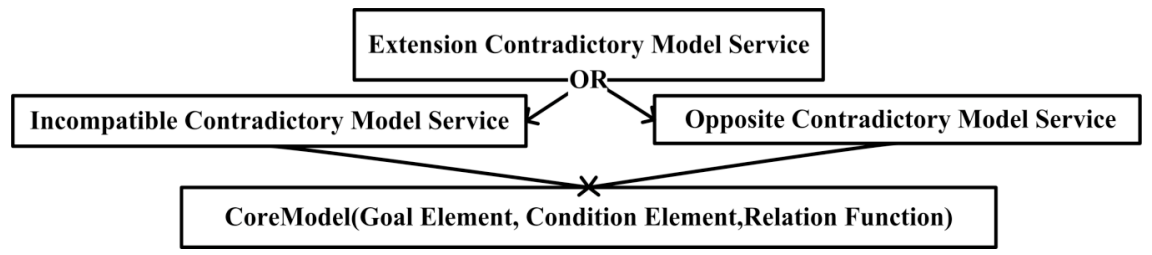

Fig. (3). Extension contradictory model service tree.

element intelligent service component, Extenics contradictory models service, as well as extensic optimization service for calculating dependent functions values. Dependent functions calculating are divided into elementary dependent function service (PrimaryDependentFunction), a simple dependent functions service (Simple Dependent Function), and discrete dependent functions service (Discrete Dependent Function), and elementary dependent function is divided into most advantages in interval midpoint (PDF_Mid), or most advantages in interval left (PDF_Left) or most advantages in interval right end (PDF_Right) three services. SetX (), SetA (), SetB (), SetC () and SetD () five services for set interval set of endpoint, CalculDF() service provides conditions property value and goal property value of dependent function. Place service values is calculated according to the nested intervals, which is called by Primary Dependent Function_mid service, Primary Depentent Function_Left service, Primary Dependent Function_right service. Distance service based on nested intervals to calculate the distance. Left Side Distance service based on nested intervals to calculate the left distance, Right Side Distance service based on nested intervals to calculate the right distance. Simple dependent functions service ise divided into simple dependent functions of the domain is a limited area (SDF_limited) and domain for unlimited region of simple functions (SDF_Limitless). SetX (), SetA () and SetB (), SetM () method four services, CalculRF () method is used to provide conditions property value and goal property value. Discrete dependent function service only provides CalculRF () service and implementation to the specific application. The dependent function service tree as shown in (Fig. 2).

\subsection{Extension Contradictory Model Service Tree}

Extenics contradictory model service is a judgment about conflict between goals and conditions. By passing the goals basic element, conditions basic element and dependent functions, and calculate and determine the conflict exists or not. Extenics contradictory model service divided into incompatible contradictory model services (Incompatible Contradictory Model Service) and antithetical contradictory model services (Antithetical Contradictory Model Service) in two categories, they are called common core model CoreModel (Goal Element, Condition Element, Dependent Function), the parameter is the goals element, the conditions element and the dependent functions. The core model service uses dependent function to calculus associated value for determining whether it is compatible (coexistence), then feedback the results to the user. The Extension contradictory model service tree as shown in (Fig. 3).

\subsection{Extension Analysis Services Tree}

The extensible analysis services mainly provide extension reasoning mechanism, includes divergence (Divergence Analysis Service), correlative (Correlative Analysis Service), implication (Implication Analysis Service), opening up(Opening up Analysis Service), conjugate (Conjugate Analysis Service) and so on. Divergence analysis service is divided into attribute values (Value_DA), attribute names and attribute values (Attribute Value_DA), basic element name (Element_DA), basic element name and attribute values (Element Value_DA) as well as basic element name and attribute name (ElementAttribute_DA) and so on five divergence analyses services. Opening up analysis service is divided into 


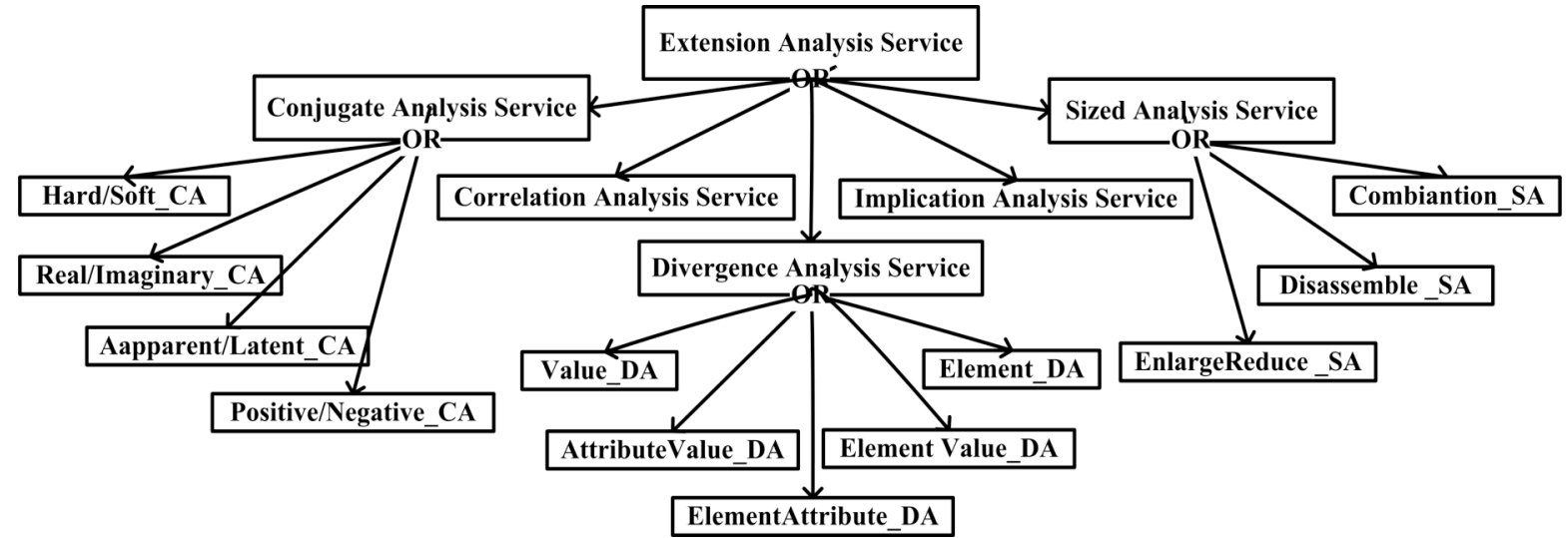

Fig. (4). Extension analysis service tree.

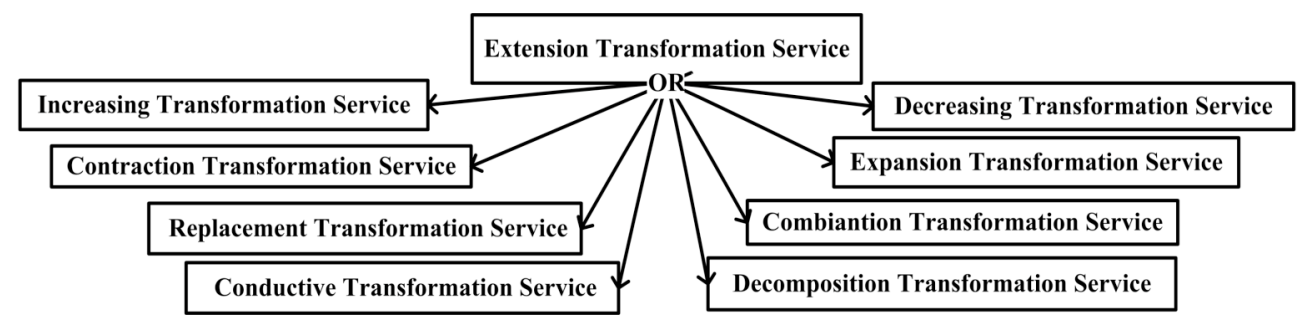

Fig. (5). Extension transformation service tree.

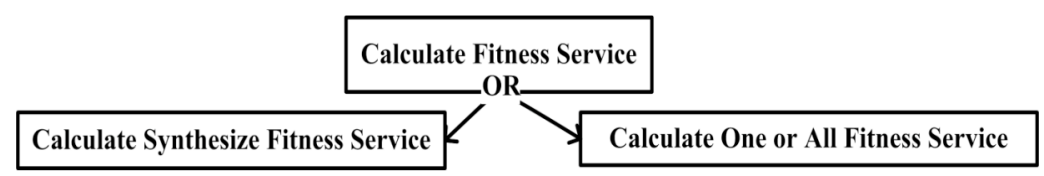

Fig. (6). Calculate fitness service tree.

combinable (Combinable_OA), disassemble (Disassemble $\mathrm{OA})$, enlarge (Enlarge_OA $)$, contract Contract_OA) and so on four opening up analysis services. Conjugate analysis service also includes hard and soft (Hard/Soft_OA), nonmaterial and material (Nonmaterial /Material OA), latent and apparent (Latent/Apparent_OA), negative and positive(Negative/Positive_OA) and so on four conjugate analysis services. Conjugate analysis service tree as shown in (Fig. 4).

\subsection{Extension Transformation Service Tree}

Extension transformation service provides transformation reasoning mechanisms, includes increasing (Increasing Transformation Service), decreasing (Decreasing Transformation Service), contraction (Contraction Transformation Service), expansion, (Expansion Transformation Service), replacement (Replacement Transformation Service), combination (Combination Transformation Service), conductive (Conductive Transformation Service), decomposition (Decomposition Transformation Service), copy (Copy Transformation Service) and so on nine transform services. Extension transformation service tree as shown in (Fig. 5).

\subsection{Calculate Fitness Service Tree}

Calculate fitness service provides evaluation about the superiority of basic element, strategy and method, etc. in Extenics. It includes calculate synthesize fitness service and calculate one or all fitness service. Calculate fitness service tree as shown in (Fig. 6).

\section{EXTENICS INTELLIGENT SERVICE SYSTEM}

According Extenics innovative methods to integrate all extension intelligent service component (EISC) which of create and innovative actives needed, should form Extenics intelligent service system(EISS). Extenics innovative software system model as shown in (Fig. 7). In the diagram, an extenics intelligent service component request service interface is connected to other intelligence services component provide service interface with a dotted line. This expresses extenics intelligent service components can dynamic connect each other on demand through a pair request and provide services, and collaborate to finish more complex service. The collaboration of Extenics intelligent service components is as follows:

1. The contradictory problem shall be abstract into goals and conditions, which are expressed respectively with the base element intelligent service component (BasicElement Intelligent Service Component) or composite element intelligent service component (CompositeElement Intelligent Service Component). They access belief information by requesting intelligent database services from/to database access intelligent service component (DataBase Access Intelligent Service Component).

2. At the same time, basic element intelligent service component (BasicElement Intelligent Service Component), or composite element intelligent service component (CompositeElement Intelligent Service Component) requests the extenics contradictory model service component 


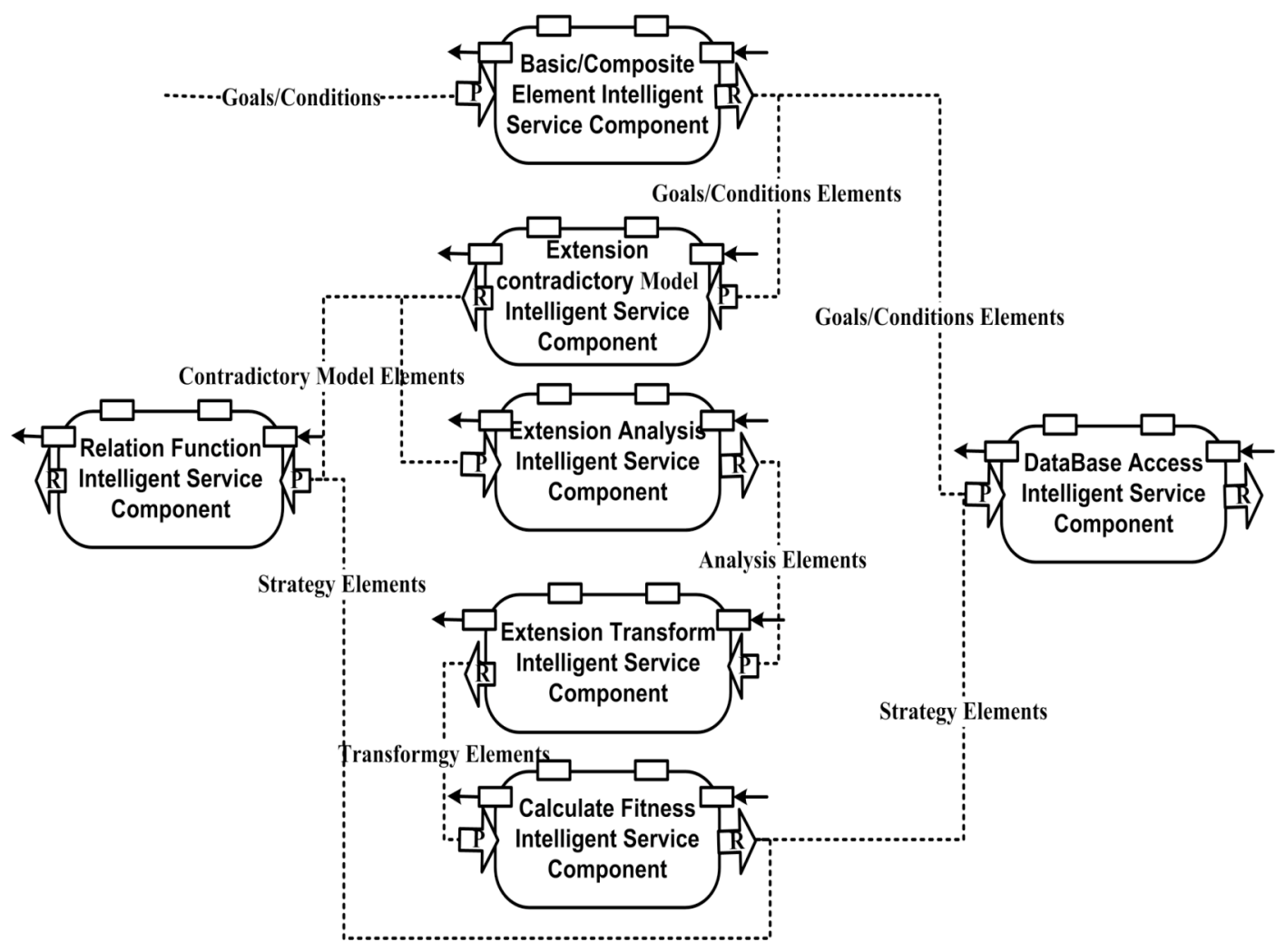

Fig. (7). Extenics intelligent service systems.

(Extenics Contradictory Model Intelligent Service Component), establish creative and innovative extenics model and its nuclear issues model.

3. Extenics contradictory model intelligent service component (Extenics Contradictory Model Intelligent Service Component) requests dependent functions intelligent service component (Dependent Function Intelligent Service Component) for calculating correlation values, and returns the calculating results.

4. Extenics contradictory model intelligent service component (Extenics Contradictory Model Intelligent Service Component) judges if is conflict or not, with return correlate values. If there is no conflict, returns related information to the user, otherwise, it requests extension analysis intelligent services components (Extension Analysis Intelligent Service Component) for seeking various solutions of contradictory problems.

5. Extension analysis intelligent services components (Extension Analysis Intelligent Service Component) uses various analysis methods to seek possible extension basic elements, and requests extenics transformation intelligent service component (Extenics Transform Intelligent Service Component) for carrying out transformation of all possible extension basic elements, formed to all possible solutions of contradictory problems.

6. Extensible transformation intelligent service component (Extension Transform Intelligent Service Component) requests the superiority evaluation intelligent service component (Superiority Evaluation Intelligent Service Component) for Extenics superiority computing services, and the superiority evaluation intelligent service component (Superiority Evaluation Intelligent Service Component) requests dependent functions intelligent service component (DependentFunction Intelligent Service Component) computing correlation values. the superiority evaluation intelligent service component (Superiority Evaluation Intelligent Service Component) evaluate the extent of each solutions to meet user requirements, and request database access intelligent service components (DataBase Access Intelligent Service Component) to access selected solutions form/to belief database for user decisionmaking.

\section{CURRENT \& FUTURE DEVELOPMENTS}

In order to help people develop intelligence, activate creativity and innovation, develop the extenics innovation software system is key. The paper presents fusion agents, services and components to integrate an autonomous, dynamic evolution, larger-granularity intelligent services component. For achieving flexibility and extensibility, we customize Extenics elements to the EISC extension intelligent service component (EISC), construct extension analysis, extension transforms, dependent functions, extension evaluation into appropriate services, through the provided services or required service approach to aggregate these Extenics intelligent service components into Extenics Intelligent Service System (EISS), and finally established an extensive innovative software system model, for modeling flexible intelligent software provide new method. In future, we shall develop cognitive function module and add into intelligent service component for improving its power of reasoning and decision-making. 


\section{CONFLICT OF INTEREST}

The authors confirm that this article content has no conflict of interest.

\section{ACKNOWLEDGEMENTS}

Declared none.

\section{REFERENCES}

[1] C. Wen, "Extension set and non-compatible problems", J. Scie. Explor", vol. 1, 1983, pp.83-97.

[2] C.Wen, Y.Chunyan and H. Bing. "Preliminary extension logic", Beijing Science Press, 2003.

[3] L. Lixi, Y. Chunyan, and L. Huawen, "Extension strategy generating system", Beijing Science Press, 2006.

[4] Y.Chunyan and C.Wen. "Extension Engineering", Beijing Science Press, 2007.

[5] C. Wen, Y. Chunyan, C. Wenwei, and L. Xingsen. "Extension set and Extension data mining", Beijing Science Press, 2008

[6] D. Jun, "Total quality management system for product life cycle modeling and realization technology research", [PhD thesis], Hangzhou, Zhejiang University, 2009.

[7] X. Ming, "Network security management technology based on Extenics", [PhD thesis], Wuhan, Normal University of Central China, 2008.

[8] Y. Yanchao, "Products that support industry collaboration modeling and application service system construction research", [PhD thesis], Chengdu, Xinan traffic University, 2009.

[9] W. Jing, "Study on description logic based on extension set". [PhD thesis], Harbin, Harbin Engineering University, 2009

[10] S. Nan, "Product configuration design based on extension logic", [PhD thesis], Hangzhou, Zhejiang University of technology, 2009.

[11] Y. Chunyan, L. Xiaomei, C. Wenwei, and C. Wen. "Extension data mining methods and its computer realization", Guangzhou. Guangdong higher education Publishing House, 2010.

[12] Y. Chunyan, L. Xingsen, "Research Progress in Extension Innovation Method and its Applications", Indus. Engr. Journal, vol. 15, no.1, pp. 131-137, 2012

[13] Y. Zhao, N. Su, P. Zhou, H. Tang, and J. Wu, "Product concept design method based on extension information matter-element", CN. Patent,.ZL200510062040.X, 12.19. 2007

[14] Y. Zhao, N. Su, L. Xing, M. Zhang, Y. Gui, J. Chen, "Dummy assembly method based on extension-able correlation relation", CN. Patent, .ZL200810062525,.06.13.2008

[15] Y. Zhao, N. Su, H. Tang, F. Zhao, J. Chen, and Y. Gui, "Part classification method based on developable clustering", CN. Patent, ZL 200810062348,05.09.2008.

[16] Y. Yu, Y. He, W. Jiang, and L. Jiang, "Non-current transformer type power-frequency weak-current electromagnetism signal sensor", CN. Patent, ZL200920054202.9, 04.08.2009

[17] Y. Yu, Y. He, W. Jiang, and L. Jiang, "Indoor wall-embedded wire nondestructive detector", CN. Patent, ZL200920054205.2, 04.09.2009.

[18] X. He, T. Li, Z. Wang, and Y. Yu, "Automatic signing machine", CN. Patent, ZL200920056631.X, 05,15,2009.

[19] N. Su, Y. Ye, Y. Zhao, and H. Wang, "Intelligent lighting control method for park landscapes based on RFID (radio frequency identification) and extension correlation functions", CN. Patent, CN201010594980.4,12.18.2010.

[20] J. Chen, Y. Gui, Z. Li, Y. Lu, Y. Ma, N. Su, Y. Ye, and Y. Zhao, "Method for resolving attribute conflicts in green design based on transforming bridge", CN. Patent, CN201010160796,04.30.2010.

[21] S. Changai, J. Maozhong, and C. Liu. "Survey of software architecture", J. Softw., vol. 13, no. 7, 2002, pp. 1229-1237.

[22] M.Shaw., "The coming-of-age of software architecture research", in Proceedings of the $23^{r d}$ International Conference on Software Engineering. Washington,DC: IEEE Computer Society, 2001, pp. 657-664.

[23] R. Allen, R. Douence, and D. Garlan, "Specifying and Analyzing
Dynamic Software Architectures", Proceedings on Fundamental Approaches to Software Engineering, Lisbon, Portugal, March 1998, LNCS 1382, pp. 21-37.

[24] R. Hongmin, "Formalization of software architecture research based on Pi calculus", [PhD thesis], Fudan University, Shanghai, 2003.

[25] L. Changyun, "Study on architecture-based software evolution", [PhD thesis], Zhejiang University, Hangzhou, 2005.

[26] G. Hongquan, "Based on formal semantics of component composition and evolution of research", [PhD thesis], Fudan University, Shanghai, 2005.

[27] R. Milner, "The Space and Motion of Communicating Agents", Cambridge University Press, 2009.

[28] C. Zhiming, "Study on the key technology of adaptive software architecture of the system", [PhD thesis], Changsha, National defense Science and Technology University, 2009.

[29] J. longfei, "Study on formal ontology engineering", [PhD thesis], Jilin, Jilin University, 2008.

[30] H. Wei, "Category", Beijing science press. 2006.

[31] H. Jinkui, "Formal semantic architecture supporting model-driven development and transformation research on consistency", $[\mathrm{PhD}$ thesis], Jinan, Shandong University, 2008.

[32] W. Qingquan, "Emergency decision based on the category of knowledge study on the matching of supply and demand", $[\mathrm{PhD}$ thesis], Dalian, Dalian University, 2009.

[33] Q. Yanwen, "Software Behaviour", Beijing, Publishing house of electronics industry. 2004.

[34] Y. Fuqing, and M. Hong. "Interware: new software forms in the future", Education network in China, 2005, pp. 52-54.

[35] L. Jian, M. Xiaoxing, T. xianping, X. Feng, and H. Hao, "Research and development of Interware", Science in China series einformation science, vol. 36, no. 10, 2006, pp. 1037-1080.

[36] Y. Jie, "Interware based on software architecture research on assembling technology", [PhD thesis], Peking University, Beijing, 2007.

[37] H. Keqing, P. Rong, L. Wei, W. Jian, and L. Bing. Network software. Beijing science press. 2008.

[38] H. Keqing, M. Yutao, L. Qing, L. Bing and P. Rong, "Software Network", Beijing science press. 2008

[39] H. Keqing, H. Yangfan, W. Shun, L. Peng and L. Jin, “ Ontology modeling theory, methods and their applications", Beijing science press. 2008.

[40] D. Weyns, and J. P. Muller, "Agent-Oriented Software Engineering $X I I^{\prime}$, The $12^{\text {th }}$ International Workshop, AOSE 2011, Taipei, May 2011, Revised Selected Papers. Lecture Notes in Computer Science, Vol. 6938, Springer 2012.

[41] D. Weyns, and M. P. Gleizes, "Agent-Oriented Software Engineering $X T^{\prime}, 11^{\text {th }}$ International Workshop, AOSE 2010, Toronto, Canada, May 10-11, 2010, Revised Selected Papers. Lecture Notes in Computer Science, Vol. 6788 .Springer 2011.

[42] M. P. Gleizes, and J. J. Gomez-Sanz, "Agent-Oriented Software Engineering $X^{\prime}, 10^{\text {th }}$ International Workshop, AOSE 2009, Budapest, Hungary, May 11-12, 2009, Revised Selected Papers. Lecture Notes in Computer Science, Vol. 6038, Springer 2011.

[43] M. Luck, and J. J. Gómez-Sanz, "Agent-Oriented Software Engineering $I X^{\prime}, 9^{\text {th }}$ International Workshop, AOSE 2008, Estoril, Portugal, May 12-13, 2008, Revised Selected Papers. Lecture Notes in Computer Science 5386 Springer 2009.

[44] M. Luck, and L. Padgham, "Agent-Oriented Software Engineering VIII", $8^{\text {th }}$ International Workshop, AOSE 2007, Honolulu, HI, USA, May 14, 2007, Revised Selected Papers. Lecture Notes in Computer Science 4951 Springer 2008.

[45] L. Padgham, and F. Zambonelli, "Agent-Oriented Software Engineering VII", $7^{\text {th }}$ International Workshop, AOSE 2006, Hakodate, Japan, May 8, 2006, Revised and Invited Papers. Lecture Notes in Computer Science 4405 Springer 2007

[46] Z. Jie, G. Liang, and L. Peigen, "Application of multi Agent technology in advanced manufacturing", Science Publishing House, 2004

[47] D. Hongxia, "Research of Web service composition based on Agent technology", [PhD thesis]., Shanghai Jiao Tong University, Shanghai, 2009.

This is an open access article licensed under the terms of the Creative Commons Attribution Non-Commercial License (http://creativecommons.org/licenses/by$\mathrm{nc} / 3.0 /$ ) which permits unrestricted, non-commercial use, distribution and reproduction in any medium, provided the work is properly cited. 